\title{
Epithelial cell adhesion molecule (EpCAM) is involved in prostate cancer chemotherapy/radiotherapy response in vivo
}

\author{
Jie Ni ${ }^{1,2}$, Paul Cozzi ${ }^{2,3}$, Julia Beretov ${ }^{1,2,4}$, Wei Duan ${ }^{5}$, Joseph Bucci ${ }^{1,2}$, Peter Graham ${ }^{1,2}$ and Yong $\mathrm{Li}^{1,2,6^{*}}$
}

\begin{abstract}
Background: Development of chemo-/radioresistance is a major challenge for the current prostate cancer (CaP) therapy. We have previously demonstrated that epithelial cell adhesion molecule (EPCAM) is associated with CaP growth and therapeutic resistance in vitro, however, the role of EPCAM in CaP in vivo is not fully elucidated. Here, we aimed to investigate how expression of EpCAM is involved in CaP growth and chemo-/radiotherapy response in NOD/SCID mouse models in vivo and to validate its role as a therapeutic target for CaP therapy.

Methods: EpCAM was knocked down in PC-3 CaP cell line using short hairpin RNA (shRNA). The effect of EpCAMknockdown (KD) on tumour growth, chemo-/radiotherapy response and animal survival was evaluated on subcutaneous (s.c) and orthotopic mouse models.

Results: We found that KD of EpCAM significantly inhibited tumour growth, increased xenograft sensitivity to chemotherapy/radiotherapy, and prolonged the survival of tumour-bearing mice. In addition, we demonstrated that KD of EpCAM is associated with downregulation of the PI3K/Akt/mTOR pathway.

Conclusions: In conclusion, our data confirms that CaP growth and chemo-/radioresistance in vivo is associated with over-expression of EpCAM, which serves both a functional biomarker and promising therapeutic target.
\end{abstract}

Keywords: EpCAM, Prostate cancer, Animal model, PI3K/Akt/mTOR signaling pathway, Chemoresistance, Radioresistance

\section{Background}

Prostate cancer $(\mathrm{CaP})$ is the commonly diagnosed and the second leading cause of cancer-related death in males in the USA in 2017 [1]. Thanks to the radical prostatectomy and radiotherapy (RT) patients with localized $\mathrm{CaP}$ can achieve excellent long-term survival. However, for recurrent and metastatic diseases, while initially responsive to androgen deprivation therapy, recurrent castration-resistant prostate cancer (CRPC) will inevitably occur, and is often associated with a poor prognosis.

RT continues to be one of standard treatment options for the different stages of CaP. Despite recent advances

\footnotetext{
*Correspondence: y.li@unsw.edu.au

${ }^{1}$ Cancer Care Centre, St George Hospital, Level 2, 4-10 South St, Kogarah, NSW 2217, Australia

${ }^{2}$ St George and Sutherland Clinical School, Faculty of Medicine, UNSW

Sydney, Kensington, NSW 2052, Australia

Full list of author information is available at the end of the article
}

in the approaches of delivering radiation to the prostate, around $30-50 \%$ of patients who undergo RT will experience biochemical recurrence within 10 years following treatment [2], which highlights the importance of understanding the biological mechanisms underlying radioresistance in attempts to predict patient response to RT prior to treatment and to identify novel therapeutic targets to overcome $\mathrm{CaP}$ radioresistance.

Despite the revolutionary targeted therapies and immunotherapy in $\mathrm{CaP}$ management, chemotherapy retains a key role in the treatment of advanced $\mathrm{CaP}$ disease. Several recent clinical trials showed profound benefits with docetaxel (DTX) by prolonging the survival, at the cost of significant toxicity [3, 4]. DTX plus prednisone regimens has been the first-line chemotherapy since 2014, however, not much progress has been made in an effort to further improve the outcome for CRPC patients [5]. Hence, it is

(c) The Author(s). 2018 Open Access This article is distributed under the terms of the Creative Commons Attribution 4.0 International License (http://creativecommons.org/licenses/by/4.0/), which permits unrestricted use, distribution, and reproduction in any medium, provided you give appropriate credit to the original author(s) and the source, provide a link to the Creative Commons license, and indicate if changes were made. The Creative Commons Public Domain Dedication waiver (http://creativecommons.org/publicdomain/zero/1.0/) applies to the data made available in this article, unless otherwise stated. 
important to uncover the key mechanisms of DTX resistance of $\mathrm{CaP}$ and identify effective therapeutic targets to improve current chemotherapeutic modalities.

Epithelial cell adhesion molecule (EpCAM), also known as CD326, is a transmembrane glycoprotein that is highly expressed in rapidly proliferating carcinomas [6]. Recent data suggest that EpCAM plays an important role not only in cell-cell adhesion, but also in cell signalling, migration, proliferation and differentiation [7]. Our previous study also demonstrated that over-expression of EpCAM was found in primary $\mathrm{CaP}$ tissues and lymph node metastases and is positively associated with $\mathrm{CaP}$ metastasis and therapeutic resistance in vitro [8]. However, little work has been done to elucidate the roles of EpCAM in $\mathrm{CaP}$ chemo - /radioresistance in vivo. To address this unmet need, in the current study, the effect of EpCAM on CaP growth and chemo-/radiotherapy response was assessed by testing the tumor growth rate of $\mathrm{CaP}$ subcutaneous (s.c.) and orthotopic xenografts on NOD/SCID mice. We demonstrate for the first time that EpCAM is involved in tumour growth, chemo-/radiotherapy response and associated with activation of the PI3K/Akt/mTOR signalling pathway in $\mathrm{CaP}$ animal models in vivo. Our results suggest that EpCAM is not only an important functional biomaker in $\mathrm{CaP}$ development and therapeutic sensitivity, but also a promising therapeutic target to repress $\mathrm{CaP}$ growth and overcome the resistance to chemo-/radiotherapy.

\section{Materials}

\section{Antibodies and reagents}

Detailed information and conditions for all antibodies are listed in Additional file 1: Table S1. DTX was purchased from Hospira Australia Pty Ltd. (Melbourne, VIC, Australia). For in vitro study, DTX was first diluted in $100 \%$ ethanol and then added to the growth medium. For in vivo study, DTX was diluted in $0.9 \%$ saline.

\section{Cell line and cell culture}

PC-3 CaP cell line was purchased from American Type Culture Collection (Rockville, MD, USA) and cultured in RPMI-1640 supplemented with $10 \%$ (vol/vol) fetal bovine serum (FBS), $50 \mathrm{U} / \mathrm{mL}$ of penicillin, and $50 \mu \mathrm{g} / \mathrm{mL}$ of streptomycin. All cell culture reagents were supplied by Life Technologies Australia Pty Ltd. (Mulgrave, VIC, Australia) unless otherwise stated. The cell line was maintained in a humidified incubator at $37^{\circ} \mathrm{C}$ and $5 \% \mathrm{CO}_{2}$.

The identity of cell lines was confirmed by short tandem repeat profiling and experiments were carried out within a limited number of passages of initial authentication.

\section{shRNA transfection for EpCAM}

Knockdown (KD) of EpCAM were achieved using a previously published method with modification [9]. Three MISSION ${ }^{\circ}$ lentiviral transduction particles encoding for
shRNA against EpCAM and MISSION ${ }^{\circ}$ non-target shRNA control transduction particles were used (Sigma-Aldrich, Pty Ltd., Castle Hills, NSW, Australia). After transfection, the stable clones were selected in cell culture medium containing $0.5 \mathrm{mg} / \mathrm{mL}$ puromycin (Invitrogen Australia Pty Ltd., Melbourne, VIC, Australia), and propagated for the following experiments.

\section{Immunofluorescence (IF) staining}

Cells grown on Millicell ${ }^{\circ}$ EZ slides (Merck Millipore, VIC, Australia) were fixed by cold methanol, rinsed by TBS (pH 7.5), blocked in 10\% (vol/vol) goat serum, and incubated with rabbit anti-human EpCAM antibody at $4{ }^{\circ} \mathrm{C}$ overnight $(\mathrm{o} / \mathrm{n})$, and then incubated with Alexa Fluro $^{\circ} 488$ goat anti-rabbit IgG $(\mathrm{H}+\mathrm{L})$ secondary antibody for $1 \mathrm{~h}$ at room temperature $(\mathrm{r} / \mathrm{t})$. Negative controls were treated identically, but using non-specific rabbit IgG. Propidium iodide (PI) was used for nuclei staining.

Immunofluorescence staining was then visualised using a ZEISS Axio Vert.A1 microscope (Carl Zeiss Microscopy, Germany).

\section{Western blot}

Western blot analysis was performed as previously described [9]. Briefly, $20 \mu \mathrm{g}$ whole cell lysates were separated on a Bis-Tris gel and then transferred to a polyvinylidene difluoride (PVDF) membrane. The membrane was blocked with $5 \%$ bovine serum albumin (BSA) in Tris-buffered saline with $0.1 \%$ Tween 20 (TBS-T), and then incubated with primary antibodies (Additional file 1: Table S1) at $4{ }^{\circ} \mathrm{C}$ $\mathrm{o} / \mathrm{n}$. Following incubation with HRP-conjugated secondary antibodies, immunoblot was analysed using SuperSignal West Pico enhanced chemiluminescence (ECL) substrate (Pierce Chemical Co, Rockford, USA) [9]. Mouse anti-human $\beta$-tubulin monoclonal antibody (MAb) was used as a loading control.

\section{Quantitative real-time PCR (qRT-PCR)}

Total RNA was isolated with High Pure RNA isolation kit (Roche Life Science, NSW, Australia) and $2 \mu \mathrm{g}$ of total RNA was used to synthesise cDNA using the SuperScript III First-strand Synthesis System Kit (Invitrogen Pty Ltd., VIC, Australia), according to the manufacturers' protocol. qRT-PCR was carried out on a Rotor-Gene instrument (Corbett Life Science, NSW, Australia) in a solution containing SYBR ${ }^{\circ}$ Select Master Mix (Life Technologies Pty Ltd., VIC, Australia), primer and cDNA in a final volume of $20 \mu \mathrm{L}$. GAPDH was used as a reference control.

\section{Experimental animals}

All animal care and experimentation procedures followed the guideline according to protocols approved by the Animal Care and Ethics Committee (ACEC 14/46A) of UNSW 
Sydney and conformed to the Animal Research Act 1985 and Australian code for the care and use of animals for scientific purposes 8th edition (2013).

Male, 6-8 weeks old NOD/SCID mice of a weight of 15-20 g (Animal Resources Centre, WA, Australia) were housed in a group of 3 or 4 , in specific pathogen-free facilities and all experiments were performed in a laminar flow cabinet in the morning of the day unless stated otherwise. House and husbandry conditions were a 12:12 light:dark cycle, mouse maintenance food and water. Environmental enrichment included bedding, one red tinted guinea pig hut and one handful of paper wool.

Mice were kept at least 1 week before experimental manipulation. All mice were monitored daily for health status and weight were recorded at least twice a week. All mice remained healthy and active during the experiments.

Establishment of s.c. and orthotopic xenograft animal models To establish s.c. xenograft model, as described previously $[9,10]$, mice were randomised into two groups, and $2 \times 10^{6}$ PC-3-EpCAM-KD or PC-3-EpCAM-scr CaP cells in $100 \mu \mathrm{L}$ Dulbecco's phosphate-buffered saline (DPBS) were injected subcutaneously in the right rear flank region of mice, respectively. Based on our previous study and a Power calculation, tumour progression of 10 mice in each cohort $(n=10)$ was measured weekly using callipers, and tumour volumes were calculated as $0.5 \times$ (length $\times$ width $^{2}$ ) (in millimetres) for up to 8 weeks. Upon sacrifice, tumour xenografts were harvested for histological examination and immunohistochemistry (IHC).

To establish orthotopic xenograft model, mice were randomised into two groups, and $1 \times 10^{6} \mathrm{PC}-3$-EpCAM-KD or PC-3-EpCAM-scr CaP cells suspended in $50 \mu \mathrm{L}$ DPBS were injected into the prostatic lobe after exposure through a lower midline laparotomy incision. Starting from the second week after cell inoculation, based on our previous study and a Power calculation, tumour progression of mice in each cohort $(n=10)$ was monitored weekly by 3D ultrasound as described in the previous publication [11]. Upon sacrifice, primary tumours were harvested for histological examination and IHC.

\section{DTX toxicity study in NOD/SCID mice without tumours}

The dose-tolerance relationship was examined in 6 NOD/SCID mice/per group without tumours following a single i.p. administration of DTX, or saline as VC treatment respectively to determine the maximum tolerance dose (MTD). The candidate doses of DTX were 10, $20,30,40,50 \mathrm{mg} / \mathrm{kg}$, based on our previous publication [9]. The MTD was the dose at one level below when $1 / 3$ of the mice reach the endpoints that were defined in the monitoring criteria.

\section{DTX response in two CaP animal models}

When average tumour size reached $70 \pm 10 \mathrm{~mm}^{3}$ in s.c. model in each subgroup, 4 mice/per group $(n=4)$ including PC-3-EpCAM-KD and PC-3-EpCAM-scr cell lines were treated with $50 \mathrm{mg} / \mathrm{kg}$ DTX (MTD) once by i.p. injection or with VC (saline), respectively. Drugs and vehicle administration was randomised. Tumour growth was plotted by measurements using callipers as previously published [9].

When average tumour size reached $50 \pm 10 \mathrm{~mm}^{3}$ in orthotopic model in each subgroup, 4 mice/per group $(n=$ 4) including PC-3-EpCAM-KD and PC-3-EpCAM-scr cell lines were treated with $50 \mathrm{mg} / \mathrm{kg}$ DTX once by i.p. injection or with VC (saline), respectively, based on the pilot study. Drugs and vehicle administration was randomised. Tumour growth was monitored by ultrasonography and calculated using Vevo ${ }^{\circ}$ Lab 1.7.0 software (VisualSonics Inc., Ontario, Canada) according to our previously published method [11].

\section{Radiation tolerance study in NOD/SCID mice without tumours}

The tolerance study was also performed to determine the MTD in mice for RT. The dose-tolerance relationship was examined in NOD/SCID mice without tumours for local radiation treatment. Mice were anaesthetised with $50-80 \mathrm{mg} / \mathrm{kg}$ ketamine and $3-5 \mathrm{mg} / \mathrm{kg}$ xylazine (i.p.) and immobilised in a custom-designed chamber with a lead shield covering the upper half of their bodies. Mice were irradiated in the X-RAD 320 Biological Irradiator (Precision X-Ray, North Branford, CT, USA) with only lower abdomen areas being exposed. Two mice were used in each group. Mice in each group received a dose of 2 Gy/day for 2, 3, 4 and 5 consecutive days (accumulatively 4, 6, 8, 10 Gy), respectively. A sham control (SC) radiation group was also included. The MTD for RT chosen for subsequent studies was the highest dose without the exhibition of endpoints that were defined in the animal monitoring criteria.

\section{Radiation response in two CaP animal models}

When average tumour size reached $70 \pm 10 \mathrm{~mm}^{3}$ in s.c. model in each subgroup, 4 mice/per group $(n=4)$ including PC-3-EpCAM-KD and PC-3-EpCAM-scr cell lines were irradiated at a dose of 2 Gy for 4 consecutive days (a total of $8 \mathrm{~Gy}$ ) or while 4 control mice/per group $(n=4)$ went through the same procedure with the SC irradiation. Irradiation and sham irradiation exposure was randomised.

When average tumour size reached $50 \pm 10 \mathrm{~mm}^{3}$ in orthotopic model in each subgroup, 4 mice/per group $(n=$ 4) including PC-3-EpCAM-KD and PC-3-EpCAM-scr cell lines were irradiated at a dose of 2 Gy for 4 consecutive days (a total of $8 \mathrm{~Gy}$ ) or with SC (no irradiation). Irradiation and sham irradiation exposure was randomised. 


\section{Mouse survival study in s.c model}

NOD/SCID mouse s.c. xenograft model was established using PC-3-EpCAM-KD or PC-3-EpCAM-scr CaP cells as described above. When tumour size reached $70 \pm$ $10 \mathrm{~mm}^{3}, 6$ mice/per group $(n=6)$ received no treatment (used as control), DTX treatment $(50 \mathrm{mg} / \mathrm{kg}$, i.p.), DTX$\mathrm{VC}$ treatment (saline, i.p.) radiation (2 Gy per day for 4 consecutive days, a total dose of 8 Gy) or RT-SC treatment (no radiation), respectively. Allocation of mice into each cohort was randomised.

Tumour size was documented weekly using a calliper for up to 13 weeks. Endpoints were defined in the animal monitoring criteria. Comparison of the survival of mice was made using a Kaplan-Meier curve.

\section{IHC}

Standard immunohistochemistry procedures were used to visualise biomarkers of interest as previously published [12]. Briefly, paraffin sections were de-parrafinised and rehydrated, then incubated with primary antibodies $\mathrm{o} / \mathrm{n}$ at $4{ }^{\circ} \mathrm{C}$. Slides were then incubated with HRP-conjugated secondary antibody (1:150 dilution) for $45 \mathrm{~min}$ at $\mathrm{r} / \mathrm{t}$, followed by 3,3' diaminobenzidine (DAB) substrate solution (Sigma-Aldrich, Pty Ltd., Castle Hill, NSW, Australia) containing $0.03 \%$ hydrogen peroxide (VWR International, QLD, Australia), Harris Hematoxylin (Thermo Fisher Pty Ltd., VIC, Australia) for counter-staining and Scott's bluing solution (Sigma-Aldrich Pty Ltd., NSW, Australia) for bluing. Tissue sections were washed in water, dehydrated, cleared and mounted.

\section{Assessment of immunostaining}

Staining intensity $(0-3)$ was assessed using a light microscopy (Leica, Germany). The assessment criteria were as previously reported [13], where: 0 (negative, $0-10 \%$ ); 1+ (weak, 10-45\%); 2+ (moderate, 45-70\%); 3+ (strong, $70 \%$ ) of the tumour cells stained. Blind scoring was performed independently by three experienced observers (JN, JB and YL), and the average of grades was recorded.

\section{Statistical analysis}

All numerical data were expressed as mean \pm standard deviation (SD). Data from two different groups were analysed using two-tail Student's t-test. All $P$ values were 2 -sided. $P<0.05$ was considered significant. The KaplanMeier method and logrank test were used for endpoint survival analysis. All statistical analyses were performed using the GraphPad Prism 7.02 software (GraphPad, San Diego CA).

\section{Results}

\section{KD of EpCAM in PC-3 cell line}

As shown in Fig. 1, PC-3 CaP cells were transfected with EpCAM-shRNA or control shRNA. KD of EpCAM was confirmed at both protein and mRNA levels. Western blot (Fig. 1a) and IF staining (Fig. 1b) and qRT-PCR (Fig. 1c) showed that EpCAM expression level was markedly reduced in PC-3-EpCAM-KD cells compared with PC-3-EpCAM-scr and PC-3 cell lines. No significant difference was noted between PC3-EpCAM-scr cells and PC-3 wild-type control cells in each group (Fig. 1).

\section{KD of EpCAM affects tumourigenicity in PC-3 s.c. and orthotopic xenograft mouse models}

As shown in the tumour growth graphs in Fig. 2, the weekly measurements of s.c. and orthotopic xenografts were plotted. The tumour growth curve showed a significantly reduced tumour growth rate of PC-3-EpCAM-KD xenografts compared to the PC-3-EpCAM-scr group $(n=10$; $P<0.05)$, in both s.c. and orthotopic models, respectively.

In s.c. model, all xenograft tumours $(n=10)$ became palpable from the third week after cell inoculation in PC-3-EpCAM-KD group while all xenograft tumours $(n=10)$ became palpable from the second week after cell inoculation in PC-3-EpCAM-scr group. Significant tumour growth difference was found 3 weeks post cell inoculation (Mean \pm SD: EpCAM-KD, $15.2 \pm 2.2 \mathrm{~mm}^{3}$; EpCAM-scr, $53.2 \pm 14.1 \mathrm{~mm}^{3}$; Fig. $\left.2 \mathrm{a}, P<0.001\right)$. After five weeks post cell inoculation, in PC-3-EpCAM-KD group, tumour volume was $106.4 \pm 9.5 \mathrm{~mm}^{3}$, while in PC-3-EpCAM-scr group, tumour volume was $183.6 \pm$ $18.4 \mathrm{~mm}^{3}$ (Mean \pm SD, Fig. $2 \mathrm{a}, P<0.001$ ). At the end of experiment ( 7 weeks post cell inoculation), mouse tumours were harvested, measured and weighed. In PC-3-EpCAM-KD group, tumour volume was $222.3 \pm$ $31.1 \mathrm{~mm}^{3}$, tumour weight was $0.5 \pm 0.2 \mathrm{~g}$; whereas in PC-3-EpCAM-scr group, tumour volume was $455.8 \pm$ $44.5 \mathrm{~mm}^{3}$, tumour weight was $1.6 \pm 0.1 \mathrm{~g}$ (Mean $\pm \mathrm{SD}$, Fig. $6 \mathrm{~b}, P<0.001$ in both measurement), suggesting that a significant difference exists in tumour volume and weight between the two sublines. Typical images for tumour sizes of different groups of mice in s.c model at the end of experiment are shown in Fig. 2c.

In orthotopic model, 3D ultrasound imaging was used to visualise and monitor tumour growth. The prostatic tumour became visible from the second week post cell inoculation in both PC-3-EpCAM-KD and PC-3-EpCAM-scr group. Significant tumour growth difference was found 3 weeks post cell inoculation (Mean \pm SD: EpCAM-KD, $24.7 \pm 6.0 \mathrm{~mm}^{3}$; EpCAM-scr, $41.8 \pm 4.3 \mathrm{~mm}^{3}$; Fig. $2 \mathrm{~d}$, $P<0.01)$. After five weeks post cell inoculation, in PC-3-EpCAM-KD group, tumour volume was $90.8 \pm$ $11.3 \mathrm{~mm}^{3}$, while in PC-3-EpCAM-scr group, tumour volume was $129.7 \pm 17.6 \mathrm{~mm}^{3}$ (Mean \pm SD, Fig. $2 \mathrm{~d}, P<$ 0.01 ). At the end of experiment ( 7 weeks post cell inoculation), tumours were harvested, measured and weighed. In PC-3-EpCAM-KD group, tumour volume was $187.2 \pm$ $24.9 \mathrm{~mm}^{3}$, tumour weight was $0.6 \pm 0.1 \mathrm{~g}$; whereas in 


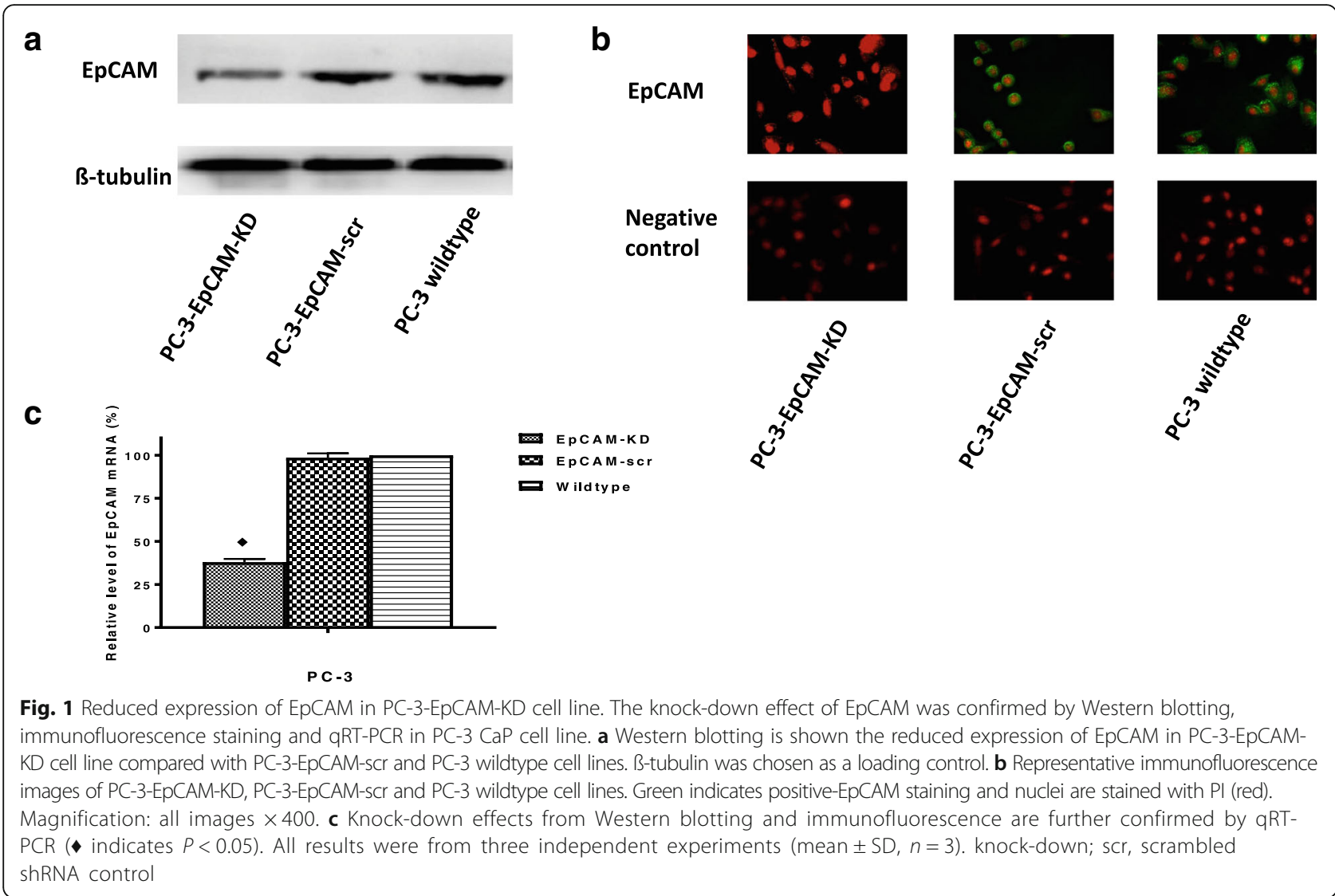

PC-3-EpCAM-scr group, tumour volume was $304.1 \pm$ $24.2 \mathrm{~mm}^{3}$, tumour weight was $1.2 \pm 0.2 \mathrm{~g}$ (Mean $\pm \mathrm{SD}$, Fig. $2 \mathrm{e}, P<0.001$ in both measurements), indicating a significant difference in tumour volume and weight in orthotopic model from the two sublines. Typical images for tumour sizes of different groups of mice in orthotopic model at the end of experiment are shown in Fig. $2 f$.

\section{KD of EpCAM increased DTX sensitivity in PC-3 s.c. and orthotopic xenograft mouse models}

In s.c. mouse model, DTX or VC treatment were administered at 4 weeks post cell inoculation. Tumour volumes were plotted against elapse time (Fig. 3a). Significant differences were seen between PC-3-EpCAM-KD-DTX and PC-3-EpCAM-KD-VC groups $(P<0.01)$, PC-3-EpCAMscr-DTX and PC-3-EpCAM-scr-VC groups $(P<0.01)$, PC-3-EpCAM-KD-VC and PC-3-EpCAM-scr-VC groups $(P<0.05)$, respectively. The ratios of DTX-treated versus VC-treated tumour volumes (DTX/VC) were calculated and plotted (Fig. 3b). Notably, two groups had different responses to DTX treatment: PC-3-EpCAM-KD xenograft responded to the DTX treatment as early as 1 week post treatment, while PC-3-EpCAM-scr started to respond to
DTX at 2 weeks post treatment. Significant differences between two groups in response to DTX were first observed at 2 weeks post DTX treatment. Overall, the ratio of PC-3-EpCAM-KD tumour volumes decreased faster in response to DTX, compared to PC-3-EpCAM-scr group $(P<0.01)$, suggesting that the KD of EpCAM can increase the DTX sensitivity of CaP tumours in s.c. mouse model. The representative images for tumour sizes at the end of experiment are shown in Fig. 3c.

In orthotopic mouse model, DTX or VC treatment was given 4 weeks post cell inoculation. Tumour volumes were plotted against elapse time (Fig. 3d). Significant differences were seen between PC-3-EpCAM-KD-DTX and PC-3EpCAM-KD-VC groups $(P<0.01)$, PC-3-EpCAM-scr-DTX and PC-3-EpCAM-scr-VC groups $(P<0.01), \quad$ PC-3-Ep CAM-KD-VC and PC-3-EpCAM-scr-VC groups $(P<0.05)$, respectively. The ratios of DTX versus $\mathrm{VC}$ tumour volumes (DTX/VC) were calculated and plotted (Fig. 3e). Overall, the ratio of PC-3-EpCAM-KD tumour volumes decreased faster in response to DTX compared to the VC group $(P<0.05)$, suggesting that the KD of EpCAM can increase the chemosensitivity of $\mathrm{CaP}$ tumours in orthotopic mouse model. The representative images of tumour sizes at the end of experiment are shown in Fig. 3f. 


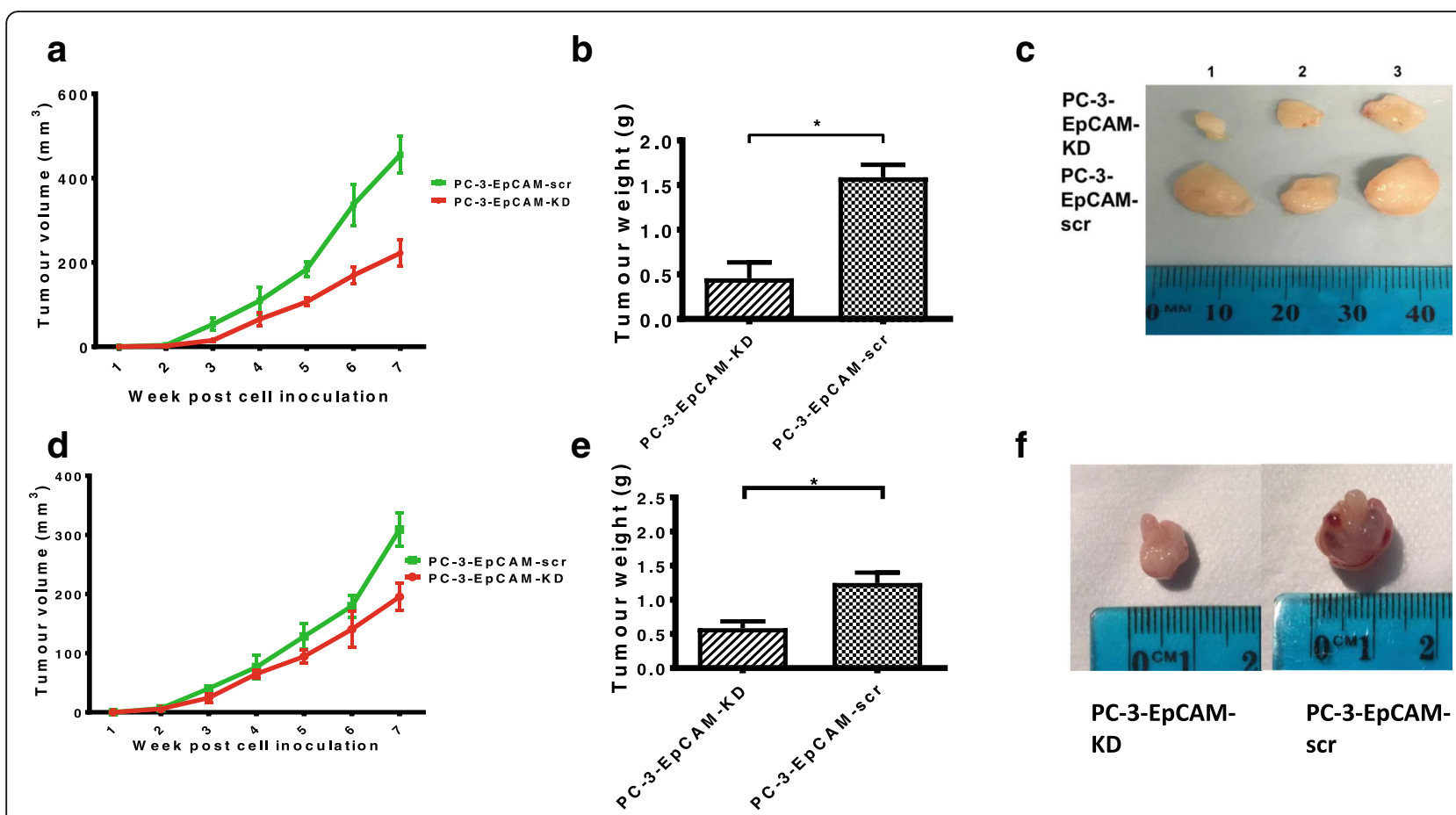

Fig. 2 Tumour growth of PC-3-EpCAM-KD and PC-3-EpCAM-scr cells in CaP S.C. and orthotopic animal models. a The graph is shown for tumour growth curves of PC-3-EpCAM-KD and PC-3-EpCAM-scr in s.C. xenograft model. The weekly measurements of PC-3-EpCAM-KD xenografts show a significant reduced tumour growth rate compared to the PC-3-EpCAM-scr group $(n=10$, mean $\pm S D, P<0.05)$. $\mathbf{b}$ At the end of the experiment, tumour weight from PC-3-EpCAM-KD group mice was significantly decreased compared to that from PC-3-EpCAM-scr group mice in s.C. model $(n=10$, mean $\pm S D$, * indicates $P<0.05)$. c At the end of the experiment, representative images of tumours from s.c. xenografts are shown. $\mathbf{d}$ The graph represents for PC-3-EpCAM-KD and PC-3-EpCAM-scr tumour growth curves in the orthotopic xenograft model $(n=10$, mean \pm SD, $P<0.05)$. e Tumour weight from PC-3-EpCAM-KD group mice was shown as significantly decreased compared to that from PC-3-EpCAM-scr group mice in orthotopic model $(n=10$, mean $\pm S D$, * indicates $P<0.05)$. $\mathbf{f}$ Representative images of tumours from orthotopic xenografts are shown for PC-3EpCAM-KD and PC-3-EpCAM-scr tumour xenografts. KD, knock-down; scr, scrambled shRNA control

\section{KD of EpCAM increased radiosensitivity in PC-3 s.c. and orthotopic xenograft mouse models}

In s.c. mouse model, RT or SC treatment was given 4 weeks post cell inoculation. Tumour volumes were plotted against elapsed time (Fig. 4a). Significant differences were seen between RT \& SC in the KD groups: PC-3-EpCAM-KD-RT and PC-3-EpCAM-KD-SC groups $(P<0.01)$, and between the scr groups: PC-3-EpCAMscr-RT and PC-3-EpCAM-scr-SC groups $(P<0.01)$, respectively. Additionally, there was a significant difference between SC treatment in KD vs scr: PC-3-EpCAMKD-SC and PC-3-EpCAM-scr-SC groups $(P<0.05)$. The ratios of RT versus SC tumour volumes (RT/SC) were calculated and plotted (Fig. 4b). Unlike the response of tumours to DTX treatment at an early stage, two groups started to respond to RT from 2 weeks post treatment, showing significant differences in response to RT which were first observed at 2 weeks post RT. Overall, as its response to chemotherapy, the ratio of PC-3-EpCAM-KD tumour volumes decreased faster in response to RT compared to the sham irradiation $(P<0.05)$, suggesting that the KD of EpCAM can increase the radiosensitivity of $\mathrm{CaP}$ tumours in s.c. mouse model. The representative images for tumour sizes at the end of experiment are shown in Fig. 4c.

In orthotopic mouse model, RT or SC treatment was given 4 weeks post cell inoculation. Tumour volumes were plotted against elapse time (Fig. 4d). Here also, significant differences were seen between RT and SC in KD and scr: PC-3-EpCAM-KD-RT and PC-3-EpCAMKD-SC groups $(P<0.01), \quad$ PC-3-EpCAM-scr-RT and PC-3-EpCAM-scr-SC groups $(P<0.05)$, as well as between SC treatment in $\mathrm{KD}$ and scr: PC-3-EpCAMKD-SC and PC-3-EpCAM-scr-SC groups $(P<0.05)$, respectively. The ratios of $\mathrm{RT}$ versus $\mathrm{SC}$ tumour volumes (RT/SC) were calculated and plotted (Fig. 4e). Starting from 3 weeks post RT, the ratio increased in PC-3EpCAM-scr group while it continued dropping in PC-3-EpCAM-KD group, suggesting that the tumour suppression effect of RT persisted longer in PC-3EpCAM-KD group. Overall, as a response to chemotherapy, the ratio of PC-3-EpCAM-KD tumour volumes decreased faster in response to RT compared to the sham irradiation $(P<0.05)$, suggesting that the KD of EpCAM 


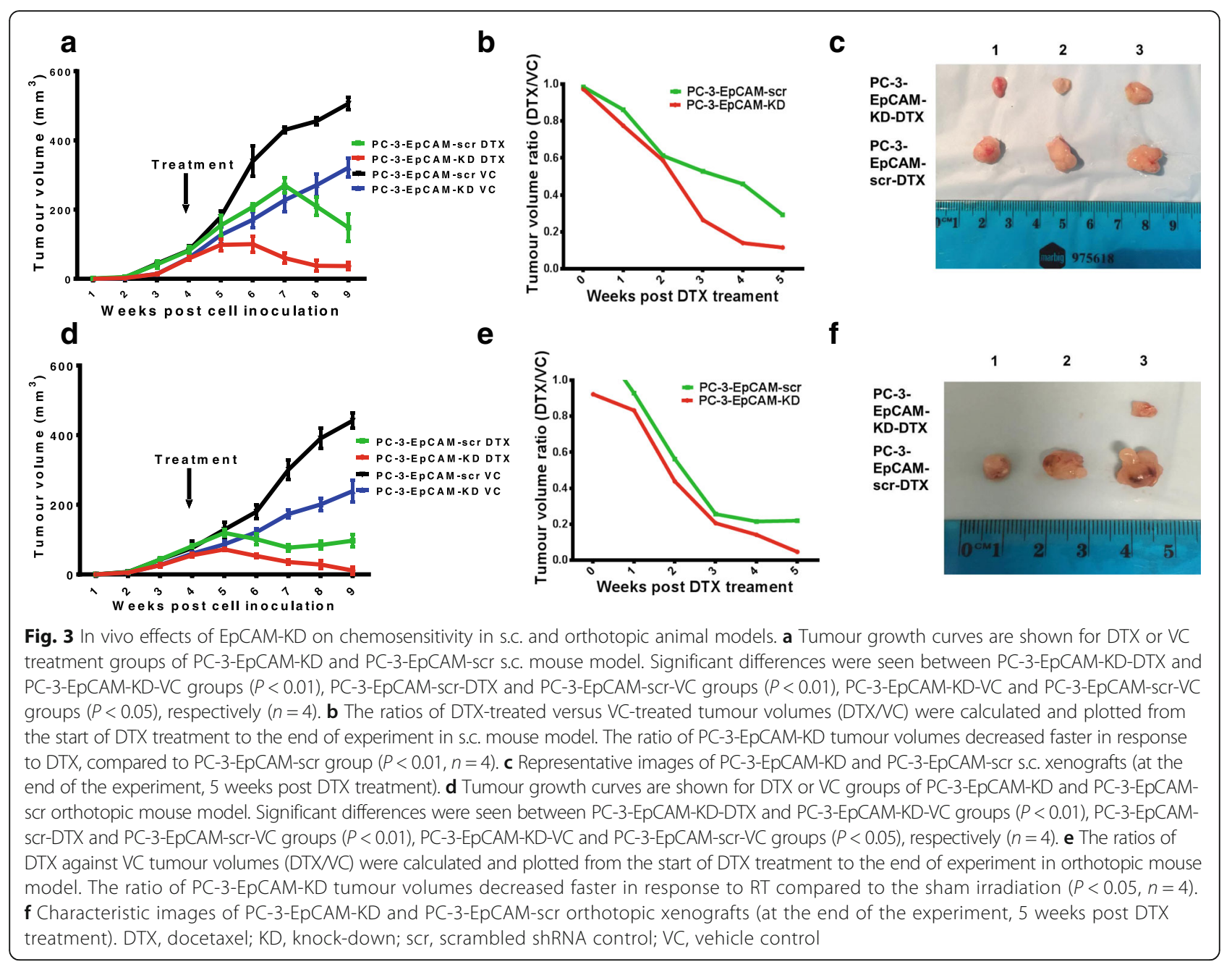

can also increase the radiosensitivity of $\mathrm{CaP}$ tumours in orthotopic mouse model. The typical images of tumour sizes at the end of experiment are shown in Fig. 4f.

\section{$\mathrm{KD}$ of EpCAM prolonged the survival of tumour-bearing mice (s.c. model) with and without treatment}

To evaluate the effect of EpCAM on tumour-bearing mice survival, Kaplan-Meier curves demonstrated that KD of EpCAM improved median survival (MS) of tumour-bearing mice (PC-3-EpCAM-KD) by 21.5 days compared with the control group mice (PC-3-EpCAM-scr s.c. xenografts) $(\mathrm{HR}=26.94, \mathrm{CI} 95 \%$ 4.317-168.1, $p=0.0004)$ (Fig. 5a). Additionally, KD of EpCAM was found to sensitise the tumour to chemo-/radiotherapy and improved MS of tumour-bearing mice which received DTX $(50 \mathrm{mg} / \mathrm{kg}$, single dose, i.p.) by 11 days ( $\mathrm{HR}=20.95$, CI95\% 3.599121.9, $P=0.0007$ ) (Fig. 5b), and RT (2Gy/day for 4 days) by 12 days $(\mathrm{HR}=11.00$, CI95\% 2.11-57.36, $P=0.0044)$ (Fig. 5c) when compared with the control group mice (PC-3-EpCAM-scr s.c. xenografts), respectively (Additional file 2: Table S2).
KD of EpCAM modulates cell proliferation, apoptosis, angiogenesis and therapeutic responses associated with the PI3K/Akt/mTOR signalling pathway

As the PI3K/Akt/mTOR signalling pathway plays a very important role in cancer therapeutic resistance including $\mathrm{CaP}[14,15]$, we investigated whether this pathway is involved in the effects of EpCAM-KD on CaP xenografts using IHC. The results demonstrated that after KD of EpCAM, along with significantly decreased expression of EpCAM, the PI3K/Akt/mTOR signalling proteins (p-mTOR and p-Akt) were also downregulated compared to EpCAMscr group, whereas no obvious changes were seen in mTOR and Akt between the two groups (Fig. 6, Additional file 3: Table S3).

To further investigate whether KD of EpCAM in CaP cells affects proliferative potential in $\mathrm{CaP}$, tumour sections from s.c. xenografts of NOD/SCID mice were assessed for proliferation using Ki-67 expression. At the end of the experiments, strong expression of Ki-67 was found in PC-3-EpCAM-scr xenograft while a weak to moderate expression of Ki-67 was observed in PC-3-EpCAM-KD 

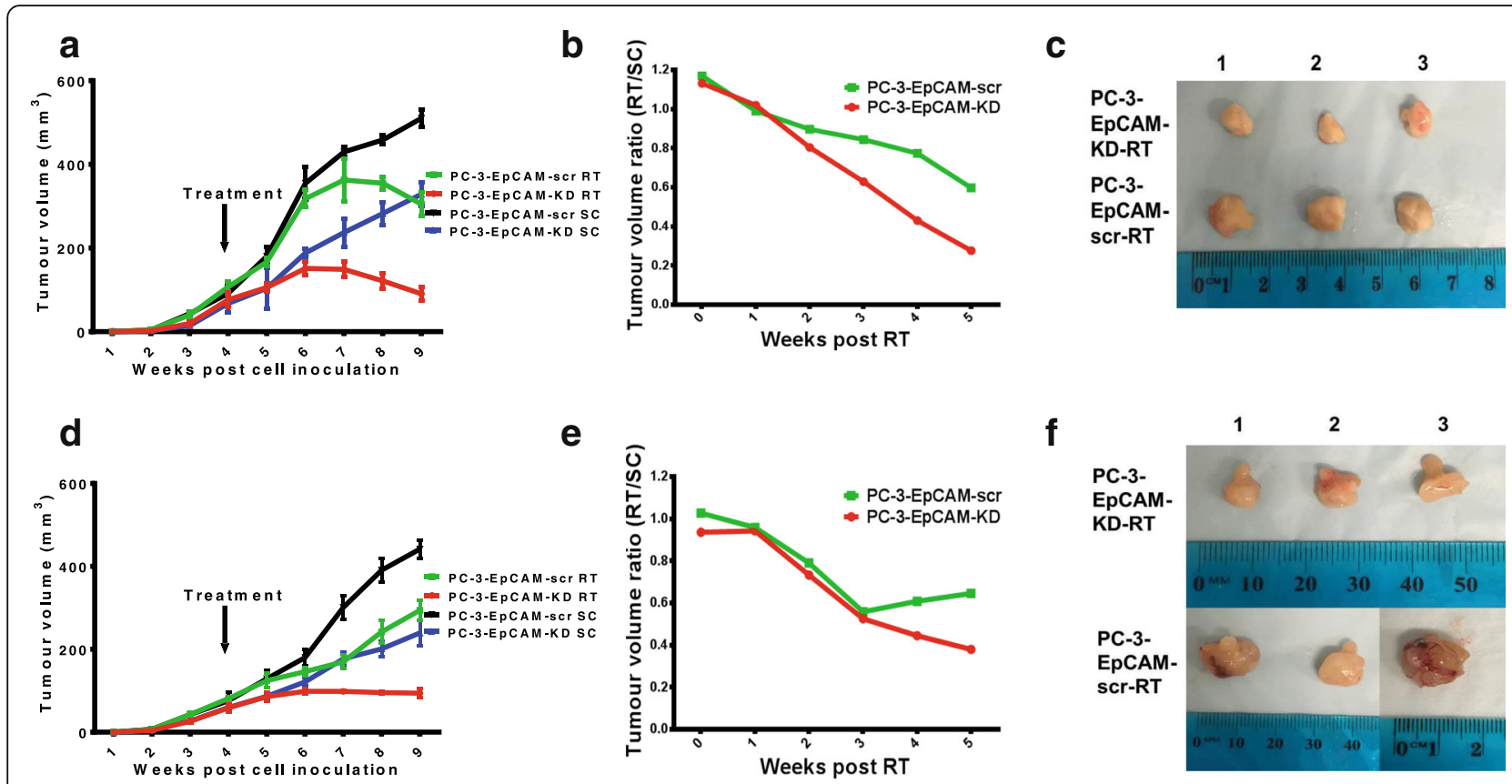

f

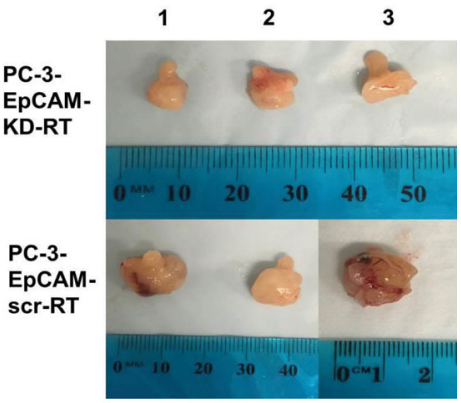

Fig. 4 In vivo effects of EpCAM-KD on radiosensitivity in S.C. and orthotopic animal models. a Tumour growth curves representing the s.c. mouse model are shown for RT or SC groups of PC-3-EpCAM-KD and PC-3-EpCAM-scr cell lines. Significant differences were seen between PC-3-EpCAMKD-RT and PC-3-EpCAM-KD-SC groups $(P<0.01)$, PC-3-EpCAM-sCr-RT and PC-3-EpCAM-scr-SC groups $(P<0.01)$, PC-3-EpCAM-KD-SC and PC-3EpCAM-scr-SC groups $(P<0.05)$, respectively $(n=4)$. $\mathbf{b}$ The ratios of RT versus SC tumour volumes $(\mathrm{RT} / \mathrm{SC})$ were calculated and plotted from the start of RT to the end of experiments in s.c. mouse model. The ratio of PC-3-EpCAM-KD tumour volumes decreased faster in response to RT compared to the sham irradiation $(P<0.05, n=4)$. c Typical images of PC-3-EpCAM-KD and PC-3-EpCAM-scr s.c. xenografts (at the end of the experiment, 5 weeks post-RT treatment). $\mathbf{d}$ Tumour growth curves representing the orthotopic mouse model are shown for RT or SC groups of PC-3-EpCAM-KD and PC-3-EpCAM-scr cell lines. Significant differences were seen between PC-3-EpCAM-KD-RT and PC-3-EpCAM-KD-SC groups $(P<0.01)$, PC-3-EpCAM-scr-RT and PC-3-EpCAM-scr-SC groups $(P<0.05)$, PC-3-EpCAM-KD-SC and PC-3-EpCAM-scr-SC groups $(P<0.05)$, respectively $(n=4)$. e The ratios of RT versus SC tumour volumes (RT/SC) were calculated and plotted from the start of RT to the end of experiments in orthotopic mouse model. The ratio of PC-3-EPCAM-KD tumour volumes decreased faster in response to RT compared to the sham irradiation $(P<0.05, n=4)$. $\mathbf{f}$ Representative images of PC-3-EpCAM-KD and PC-3-EpCAM-scr orthotopic xenografts (at the end of the experiment, 5 weeks post RT treatment). KD, knock-down; SC, sham control; RT, radiotherapy; scr, scrambled shRNA control

xenograft (Fig. 6, Additional file 3: Table S3), suggesting that EpCAM is associated with the proliferation of $\mathrm{CaP}$ in vivo.

To study the effects of KD of EpCAM on therapeutic responses, active Caspase- 3 and CD31 was examined to assess the tumour response to DTX, while $\gamma \mathrm{H} 2 \mathrm{AX}$ was examined to assess the tumour response to RT. At the end of the experiments, there was an increased Caspase-3 (active) and decreased CD31 expression found in the tumour xenografts of PC-3-EpCAM-KD CaP cells compared to PC-3-EpCAM-scr group (Additional file 3: Table S3), suggesting that increased apoptosis (active Caspase-3) and reduced angiogenic activity (CD31) were found in response to DTX treatment after KD of EpCAM. Increased $\gamma \mathrm{H} 2 \mathrm{AX}$ expression was found in tumour xenografts of PC-3-EpCAM-KD CaP cells compared to PC-3-EpCAM-scr group after RT (Additional file 3: Table S3), suggesting that sensitisation of $\mathrm{CaP}$ to $\mathrm{RT}$ could be mediated by KD of EpCAM in vivo. Representative images were shown in Fig. 6.
The staining results showed that EpCAM is associated with cell proliferation, apoptosis, angiogenesis and therapeutic responses in vivo, possibly via regulation of the PI3K/Akt/mTOR signalling pathway, suggesting that this pathway is involved in the regulation of $\mathrm{CaP}$ proliferation and chemo-/radioresistance together with EpCAM.

\section{Discussion}

Chemo-/radioresistance remains a big challenge for the current $\mathrm{CaP}$ therapy. Therapeutic resistant and refractory $\mathrm{CaP}$ cells disseminate to distant organs such as bone and liver via blood and form metastases. Up tp $30 \%$ of $\mathrm{CaP}$ patients suffered a relapse and developed a PSA recurrence, leading to a more invasive or metastatic disease within 10 years post surgery $[16,17]$. Identification of therapeutic targets that are associated with $\mathrm{CaP}$ chemo-/radioresistance is crucial for developing novel targeted therapy to overcome $\mathrm{CaP}$ therapeutic resistance. In the present study, we investigated whether EpCAM is associated with chemo-/radiotherapy response in $\mathrm{CaP}$ 
a

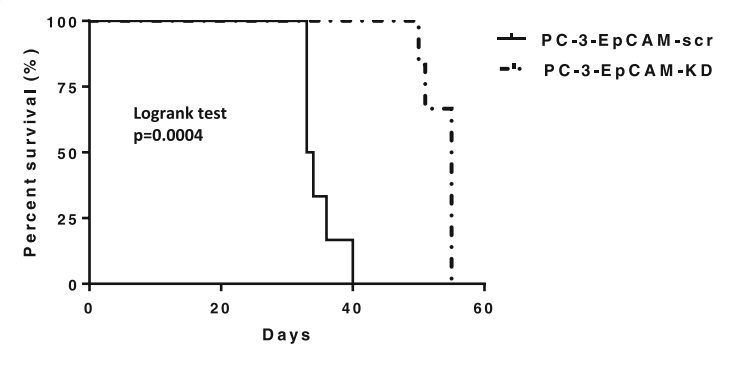

b

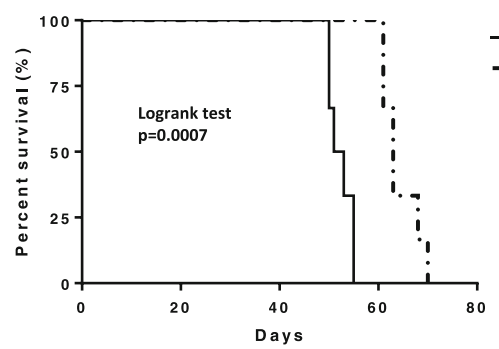

- PC-3-EPCAM-sCr DTX

C

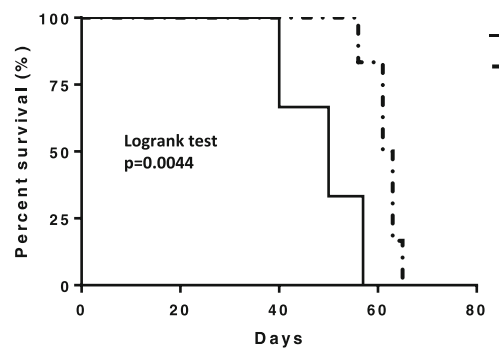

- PC-3-EPCAM-sCrRT

-1. PC-3-EPCAM-KD RT

Fig. 5 Mouse survival curves after KD of EpCAM and chemo-/radiotherapy in s.c CaP model. a Kaplan-Meier survival curves for s.c. tumourbearing mice demonstrate that KD of EpCAM prolongs mouse survival. $n=6$, logrank $p$ value is shown. $\mathbf{b}$ Kaplan-Meier survival curves for s.c. tumour-bearing mice that received DTX treatment demonstrate that KD of EpCAM prolongs mouse survival with $40 \mathrm{mg} / \mathrm{kg}$ DTX treatment. $n=6$, logrank $p$ value is shown. c Kaplan-Meier survival curves for s.c. tumour-bearing mice that received RT demonstrate that KD of EpCAM prolongs mouse survival with 2Gy RT. $n=6$, logrank $p$ value is shown. DTX, docetaxel, KD, knock-down, RT, radiotherapy

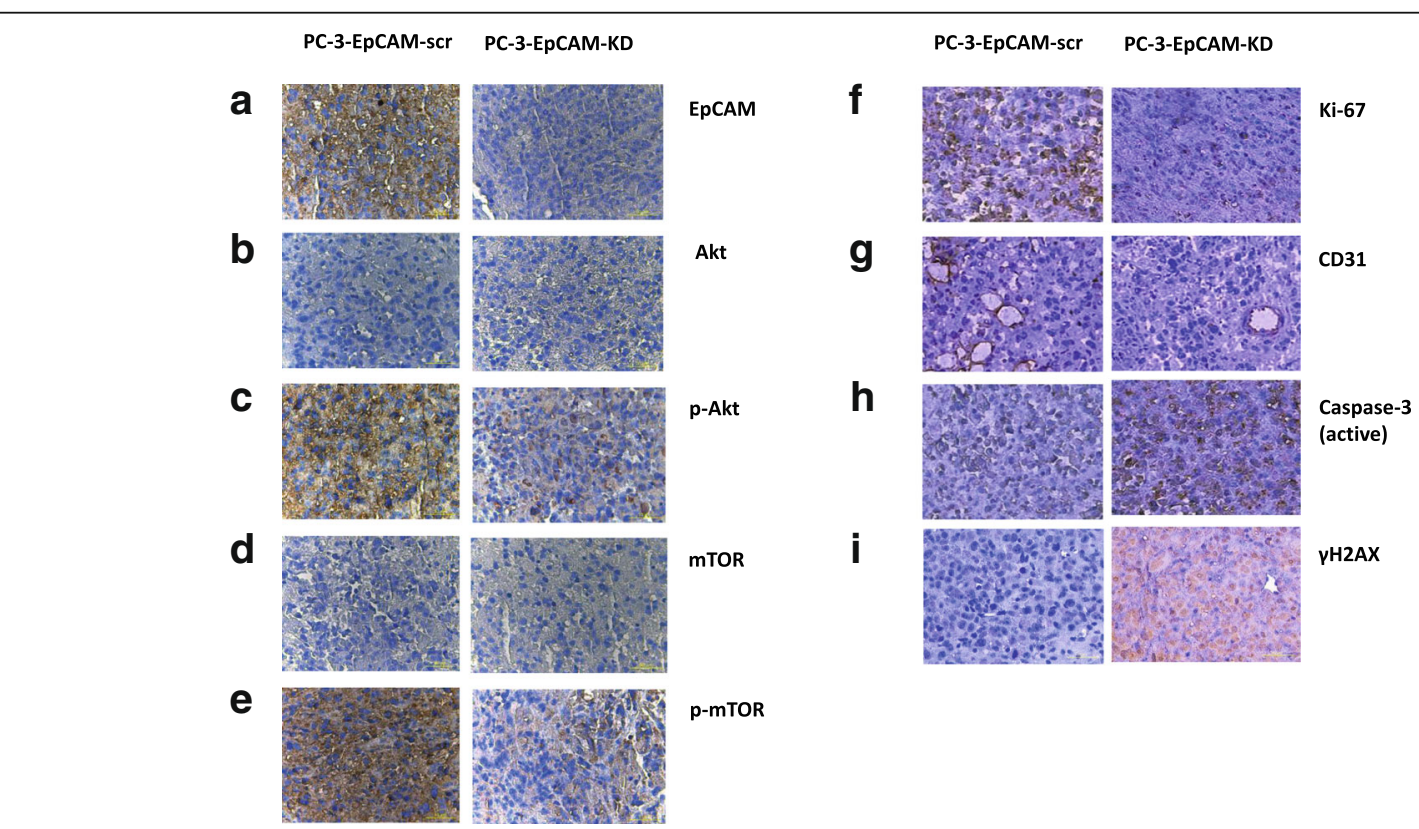

Fig. 6 Immunohistochemistry staining of EpCAM, PI3K/mTOR/Akt pathway proteins, Ki-67, CD31 Caspase-3 (active) and YH2AX expressions in s.c. mouse xenografts. a Significantly reduced expression of EpCAM was observed after KD of EpCAM in s.c. xenografts. b-e comparison of EpCAM-KD with EpCAM-scr group: PI3K/Akt/mTOR signalling proteins ( $\mathrm{p}-\mathrm{mTOR}$ and $\mathrm{p}$-Akt) were noticeably downregulated, whereas no obvious changes were seen in mTOR and Akt. $\mathbf{f}$ Strong expression of Ki-67 was observed in PC-3-EpCAM-scr s.c. xenografts while weak expression of Ki-67 was found inPC-3-EpCAM-KD xenografts. g Markedly reduced CD31 expression was seen in PC-3-EpCAM-KD xenografts compared to PC-3-EpCAM-sCr xenografts after DTX treatment. h After treatment with DTX, an increased level of active Caspase-3 expression was seen in PC-3-EPCAM-KD s.C. xenografts compared to PC-3-EpCAM-scr s.c. xenografts. i Following RT, an increased level of YH2AX expression was seen in PC-3-EpCAM-KD s.C. xenografts compared to PC-3-EpCAM-scr s.c. xenografts. Magnification $\times 400$ in all images. DTX, docetaxel; KD, knock-down; RT, radiotherapy; s.c., subcutaneous; scr, scrambled shRNA control 
animal models and a useful therapeutic target for $\mathrm{CaP}$ treatment. We first knocked down EpCAM using shRNA in PC-3 cell line and then established s.c. and orthotopic CaP mouse models using PC-3-EpCAM-KD and PC-3-EpCAMscr sublines. It was found that $\mathrm{KD}$ of EpCAM can significantly reduce tumour growth in both s.c. and orthotopic mouse models, respectively. Our data also indicated that silencing EpCAM increased prostate tumour xenograft sensitivity to chemotherapy and radiation, and significantly prolonged the survival of tumour-bearing mice. Additionally, we showed that EpCAM-regulated tumour development and treatment sensitivity is associated with activation of the PI3K/Akt/mTOR pathway. The findings of in vivo study are consistent with our recent in vitro results [6]. To the best of our knowledge, this is the first study to investigate the roles of EpCAM in CaP development and therapeutic resistance and associated signalling pathway in vivo.

As an oncogene, EpCAM promotes carcinogenesis, cell proliferation and survival, resulting in accelerated tumour growth and metastasis. In our two established xenograft animal models, IHC results also showed that reduction in proliferation (using $\mathrm{Ki}-67$ ) was associated with KD of EpCAM. Ki-67 is a human nuclear protein that is present during all active phases of the cell cycle but absent from resting cells, making it an excellent marker for measuring the proliferation of cells in human tumours [18]. Since expression of EpCAM is positively related to that of $\mathrm{Ki}-67$, EpCAM may regulate the cell proliferation via cyclin pathway. It was reported that the strength and pattern of EpCAM expression are both positively correlated with the proliferation marker Ki-67, high expression and nuclear localisation of cyclin D1, and $\mathrm{Rb}$ phosphorylation in hypopharynx carcinoma in vivo [19], further validating its role in tumorigenesis. Another model of EpCAM signaling mechanism shows that cleaved EpCAM may initiate regulated intramembrane proteolysis that the released cytoplasmic tail of EpCAM is responsible for the induction of transcription of EpCAM target genes [20].

DTX is now the most widely used and first line chemotherapeutic agent for CRPC [21]. In our study, we found that expression of EpCAM is associated with tumour responsiveness to DTX treatment in vivo. DTX achieves its anti-tumour effect by disrupting microtubule dynamics and inducing G2/M cell cycle arrest, which promotes apoptosis through the phosphorylation of antiapoptotic protein $\mathrm{Bcl}-2$ [22]. A recent study by Tayama $\mathrm{S}$, et al. provided evidence that EpCAM overexpression is an independent risk factor for chemoresistance, and correlated with a lower level of cisplatin-induced apoptosis, which is regulated by EpCAM-Bcl-2 axis in ovarian cancer [23]. The EpCAM-Bcl-2 mechanism was further validated in the same study. It was demonstrated that siRNA-mediated EpCAM KD downregulated Bcl-2 expression and upregulated Bax expression whereas transfection of EpCAM reversed the effects [23], providing a rationale for the combination therapy of EpCAM-targeting agents and conventional chemotherapy. In another study, it was found that EpCAM antibody can target $\mathrm{EpCAM}^{+}$chemoresistant leukemia subpopulations after chemotherapy in a mouse model, suggesting EpCAM is a promising novel target for the treatment of leukemia [24]. Interestingly, EpCAM was found to be significantly reduced in docetaxel-resistant PC-3 and DU145 CaP cells, as well as in prostate tissues of patients who received chemotherapeutic treatment with docetaxel [25], which our results (data not shown) were in some degree not in line with. This discrepancy can be explained by the differences in sampling (human patients versus PC-3 mouse xenografts), DOX dosages $\left(60-75 \mathrm{mg} / \mathrm{m}^{2}\right.$ versus $50 \mathrm{mg} / \mathrm{kg}$ ) [26], route of administration (intravenous versus i.p. ) and the post-treatment duration when tumour tissue was obtained (neoadjuvant settings versus 5 weeks).

Over-expression of EpCAM is also related to cancer radiosensitivity. It was demonstrated that higher incidence of intense expression of EpCAM was found in head and neck squamous cell carcinoma (HNSCC) from the hypopharynx, and patients with strong EpCAM expression were associated with local recurrence after primary RT [27]. Kaori et al. reported that down-regulation of EpCAM by siRNA increased the radiosensitivity of ME-180 cervical adenosquamous carcinoma cells [28]. Our results from the in vitro and in vivo study are consistent with Kaorie's report and indicate that down-regulation of EpCAM expression increased radiosensitivity in CaP. IHC results showed a concomitant upregulation of double-strand breaks marker $\gamma \mathrm{H} 2 \mathrm{AX}$, implying its important role in $\mathrm{CaP}$ radiosensitivity, which may help to understand the mechanism by which EpCAM regulates $\mathrm{CaP}$ radiosensitivity.

Although multiple previous studies focused on the relationship between EpCAM and survival with patients of $\mathrm{CaP}$, a consensus has not yet been reached [29-31]. In this study, Kaplan-Meier analysis was used to assess the relationship between expression of EpCAM and survival outcomes of $\mathrm{CaP}$ tumour-bearing mice. We demonstrated that KD of EpCAM prolonged the survival of tumour-bearing mice with and without chemo-/radiotherapy, suggesting that expression of EpCAM is associated with unfavourable prognosis in $\mathrm{CaP}$.

Activation of the PI3K/Akt/mTOR pathway has been shown to be actively involved in CaP progression $[8,14,32$, 33] and also important in maintenance of a CSC population [34] and promoting EMT in CaP cells [35, 36]. In this study, we have shown that permanent silencing of EpCAM, concomitantly downregulated PI3K/Akt/mTOR signalling pathway proteins and demonstrated it is associated with 
$\mathrm{CaP}$ chemo-/radiosensitivity, suggesting that the activation of this pathway is associated with EpCAM overexpression in vivo. In chemo-/radioresistant recurrent $\mathrm{CaP}$ biopsies, we found increased expression of p-AKT and p-mTOR compared with the pre-treatmentbiopsies (unpublished data), further confirming the activation of this signaling pathway in therapeutic resistant $\mathrm{CaP}$ patients.

Recent data have demonstrated a pleiotropic role of EpCAM in cancer that is not only limited to regulation of cell-cell adhesion, cell proliferation, migration and signalling [37], but also involved in CSC development and maintenance $[8,38]$, as well as circulating tumour cells (CTCs) [39]. Mounting data from recent years have indicated that EpCAM is a useful biomarker for the identification and isolation of subsets enriched for CSCs $[40,41]$, or is associated with the "stemness" in various cancers $[23,42,43]$, including $\mathrm{CaP}[8,38]$. As a result, targeting EpCAM that is responsible for sustaining prostate CSCs and developing treatment resistance might be a promising approach to effectively eradicate prostate $\mathrm{CSCs}$ as the putative root of $\mathrm{CaP}$ and $\mathrm{CaP}$ recurrence.

Based on the close association between EpCAM and $\mathrm{CaP}$ development and therapeutic responses, it will be of great clinical significance to use EpCAM in targeted therapy alone or in combination with other conventional therapies in the treatment of CRPC. Possible targeted therapies include antibodies to inactivate EpCAM proteins [44, 45], toxin-conjugated anti-EpCAM antibodies [46], immunotherapy [47], RNAi gene therapies that interfere EpCAM genes [48] as well as EpCAM-conjugated RNA aptamer [49] that can target EpCAM-positive tumour cells with superior affinity and specificity.

\section{Conclusion}

Our data have demonstrated that EpCAM is involved in $\mathrm{CaP}$ development, chemo-/radioresistance and prognosis and associated with activation of the PI3K/Akt/mTOR signalling pathway. The findings suggest EpCAM is a promising therapeutic target for overcoming $\mathrm{CaP}$ chemo - /radioresistance.

\section{Additional files}

Additional file 1: Table S1. List of antibodies used in Western Blot and immunohistochemistry. (DOCX $14 \mathrm{~kb}$ )

Additional file 2: Table S2. Survival information of the subcutaneous mouse CaP model. (DOCX $13 \mathrm{~kb}$ )

Additional file 3: Table S3. The staining intensity of various markers in subcutaneous xenografts CaP model. (DOCX $13 \mathrm{~kb}$ )

\section{Abbreviations}

Ab: Antibody; BSA: Bovine serum albumin; CaP: Prostate cancer; CRPC: Castration-resistant prostate cancer; CSC: Cancer stem cell; CTC: Circulating tumour cell; DAB: 3,3' diaminobenzidine; DPBS: Dulbecco's phosphate-buffered saline; DTX: Docetaxel; ECL: Enhanced chemiluminescence; FBS: Fetal bovine serum; HNSCC: Head and neck squamous cell carcinoma; IHC: Immunohistochemistry; KD: Knockdown; MAb: Monoclonal antibody; MTD: Maximum tolerance dose; PVDF: Polyvinylidene difluoride; RP: Radical prostatectomy; RT: Radiotherapy;

s.c.: Subcutaneous; SC: Sham control; SD: Standard deviation;

shRNA: Short hairpin RNA; VC: Vehicle control

\section{Acknowledgements}

We thank Mr. Ken Hopper from Cancer Care Centre St George Hospital for technical support in radiation, and Dr. Belamy Cheung and Dr. Daniel Carter from Children's Cancer Institute Australia for support in gene knock down.

\section{Funding}

This study was supported in part by Cancer Research Trust Fund at Cancer Care Centre, St. George Hospital; Prostate and Breast Cancer Foundation (PBCF) and Sydney Urological Research Fund (SURF).

The funding sources listed above had no role in study design; collection, management, analysis and interpretation of data; decision to publish or preparation of the manuscript.

The author(s) received no specific funding for this work.

\section{Availability of data and materials}

The datasets used and/or analysed during the current study are available from the corresponding author on reasonable request.

\section{Authors' contributions}

$J N, P C, J B u c c i, P G$ and $Y L$ designed the study framework and experiments. $\mathrm{JN}$ carried out the experiments. JBeretov contributed to the animal pathology and IHC. JN, WD, PG and YL contributed to the interpretation of the results and data analysis. JN took the lead in writing the manuscript. All authors provided constructive feedback and comments to help shape the research, analysis and manuscript. The manuscript has been read and approved by all named authors and there are no other persons who satisfied the criteria for authorship but are not listed. The order of authors listed in the manuscript has also been approved by all of us.

\section{Ethics approval and consent to participate}

The animal study was approved by the UNSW Sydney Animal Care and Ethics Committee (ACEC 14/46A). No human subjects were involved in this study.

Consent for publication

Not applicable.

Competing interests

The authors declare that they have no competing interest.

\section{Publisher's Note}

Springer Nature remains neutral with regard to jurisdictional claims in published maps and institutional affiliations.

\section{Author details \\ 'Cancer Care Centre, St George Hospital, Level 2, 4-10 South St, Kogarah, NSW 2217, Australia. ${ }^{2}$ St George and Sutherland Clinical School, Faculty of Medicine, UNSW Sydney, Kensington, NSW 2052, Australia. ${ }^{3}$ Department of Surgery, St George Hospital, Kogarah, NSW 2217, Australia. ${ }^{4}$ Anatomical Pathology, NSW Health Pathology, St George Hospital, Gray St, Kogarah, NSW 2217, Australia. ${ }^{5}$ School of Medicine and Centre for Molecular and Medical Research, Deakin University, Waurn Ponds, VIC 3216, Australia. ${ }^{6}$ School of Basic Medical Sciences, Zhengzhou University, Henan 450001, China.}

Received: 15 April 2018 Accepted: 29 October 2018

Published online: 12 November 2018

\section{References}

1. Siegel RL, Miller KD, Jemal A. Cancer statistics, 2017. CA Cancer J Clin. 2017;67(1):7-30

2. Paller $\mathrm{CJ}$, Antonarakis ES. Management of biochemically recurrent prostate cancer after local therapy: evolving standards of care and new directions. Clin Adv Hematol Oncol. 2013;11(1):14-23.

3. Tannock IF, de Wit R, Berry WR, Horti J, Pluzanska A, Chi KN, Oudard S, Theodore C, James ND, Turesson I, et al. Docetaxel plus prednisone or mitoxantrone plus prednisone for advanced prostate cancer. N Engl J Med. 2004;351(15):1502-12. 
4. Sweeney CJ, Chen YH, Carducci M, Liu G, Jarrard DF, Eisenberger M, Wong YN, Hahn N, Kohli M, Cooney MM, et al. Chemohormonal therapy in metastatic hormone-sensitive prostate Cancer. N Engl J Med. 2015;373(8):737-46.

5. Higano CS, Crawford ED. New and emerging agents for the treatment of castration-resistant prostate cancer. Urol Oncol. 2011;29(6 Suppl):S1-8.

6. Ni J, Cozzi PJ, Duan W, Shigdar S, Graham PH, John KH, Li Y. Role of the EpCAM (CD326) in prostate cancer metastasis and progression. Cancer Metastasis Rev. 2012;31(3-4):779-91.

7. Schnell U, Cirulli V, Giepmans BN. EpCAM: structure and function in health and disease. Biochim Biophys Acta. 2013;1828(8):1989-2001.

8. Ni J, Cozzi P, Hao J, Beretov J, Chang L, Duan W, Shigdar S, Delprado W, Graham P, Bucci J, et al. Epithelial cell adhesion molecule (EpCAM) is associated with prostate cancer metastasis and chemo/radioresistance via the PI3K/Akt/mTOR signaling pathway. Int J Biochem Cell Biol. 2013;45(12):2736-48.

9. Hao J, Madigan MC, Khatri A, Power CA, Hung TT, Beretov J, Chang L, Xiao W, Cozzi PJ, Graham PH, et al. In vitro and in vivo prostate cancer metastasis and chemoresistance can be modulated by expression of either CD44 or CD147. PLoS One. 2012;7(8):e40716.

10. Li Y, Song E, Abbas Rizvi SM, Power CA, Beretov J, Raja C, Cozzi PJ, Morgenstern A, Apostolidis C, Allen BJ, et al. Inhibition of micrometastatic prostate cancer cell spread in animal models by 213Bilabeled multiple targeted alpha radioimmunoconjugates. Clin Cancer Res. 2009;15(3):865-75.

11. Ni J, Cozzi P, Hung TT, Hao J, Graham P, Li Y. Monitoring prostate tumor growth in an Orthotopic mouse model using three-dimensional ultrasound imaging technique. Transl Oncol. 2016;9(1):41-5.

12. Ni J, Cozzi PJ, Hao JL, Beretov J, Chang L, Duan W, Shigdar S, Delprado WJ, Graham PH, Bucci J, et al. CD44 variant 6 is associated with prostate cancer metastasis and chemo-/radioresistance. Prostate. 2014;74(6):602-17.

13. Cozzi PJ, Wang J, Delprado W, Perkins AC, Allen BJ, Russell PJ, Li Y. MUC1, MUC2, MUC4, MUC5AC and MUC6 expression in the progression of prostate cancer. Clin Exp Metastasis. 2005;22(7):565-73.

14. Chang L, Graham PH, Ni J, Hao J, Bucci J, Cozzi PJ, Li Y. Targeting PI3K/Akt/ mTOR signaling pathway in the treatment of prostate cancer radioresistance. Crit Rev Oncol Hematol. 2015;96(3):507-17.

15. Ni J, Cozzi P, Hao J, Duan W, Graham P, Kearsley J, Li Y. Cancer stem cells in prostate cancer chemoresistance. Curr Cancer Drug Targets. 2014;14(3):225-40.

16. Roehl KA, Han M, Ramos CG, Antenor JA, Catalona WJ. Cancer progression and survival rates following anatomical radical retropubic prostatectomy in 3,478 consecutive patients: long-term results. J Urol. 2004;172(3):910-4.

17. Hull GW, Rabbani F, Abbas F, Wheeler TM, Kattan MW, Scardino PT. Cancer control with radical prostatectomy alone in 1,000 consecutive patients. J Urol. 2002;167(2 Pt 1):528-34.

18. Scholzen T, Gerdes J. The Ki-67 protein: from the known and the unknown. J Cell Physiol. 2000;182(3):311-22.

19. Chaves-Perez A, Mack B, Maetzel D, Kremling H, Eggert C, Harreus U, Gires O. EpCAM regulates cell cycle progression via control of cyclin D1 expression. Oncogene. 2013;32(5):641-50.

20. Maetzel D, Denzel S, Mack B, Canis M, Went P, Benk M, Kieu C, Papior P, Baeuerle PA, Munz M, et al. Nuclear signalling by tumour-associated antigen EpCAM. Nat Cell Biol. 2009;11(2):162-71.

21. Bracarda S, Logothetis C, Sternberg CN, Oudard S. Current and emerging treatment modalities for metastatic castration-resistant prostate cancer. BJU Int. 2011;107(Suppl 2):13-20.

22. Mollinedo F, Gajate C. Microtubules, microtubule-interfering agents and apoptosis. Apoptosis. 2003;8(5):413-50.

23. Tayama S, Motohara T, Narantuya D, Li C, Fujimoto K, Sakaguchi I, Tashiro H, Saya $\mathrm{H}$, Nagano O, Katabuchi H. The impact of EpCAM expression on response to chemotherapy and clinical outcomes in patients with epithelial ovarian cancer. Oncotarget. 2017;8(27):44312-25.

24. Zheng X, Fan X, Fu B, Zheng M, Zhang A, Zhong K, Yan J, Sun R, Tian Z, Wei $H$. EpCAM inhibition sensitizes Chemoresistant leukemia to immune surveillance. Cancer Res. 2017;77(2):482-93.

25. Massoner P, Thomm T, Mack B, Untergasser G, Martowicz A, Bobowski K, Klocker $\mathrm{H}$, Gires O, Puhr M. EpCAM is overexpressed in local and metastatic prostate cancer, suppressed by chemotherapy and modulated by METassociated miRNA-200c/205. Br J Cancer. 2014;111(5):955-64.

26. Puhr M, Hoefer J, Schafer G, Erb HH, Oh SJ, Klocker H, Heidegger I, Neuwirt H, Culig Z. Epithelial-to-mesenchymal transition leads to docetaxel resistance in prostate cancer and is mediated by reduced expression of miR-200c and miR-205. Am J Pathol. 2012;181(6):2188-201.
27. Murakami N, Mori T, Yoshimoto S, Ito Y, Kobayashi K, Ken H, Kitaguchi M, Sekii S, Takahashi K, Yoshio K, et al. Expression of EpCAM and prognosis in early-stage glottic cancer treated by radiotherapy. Laryngoscope. 2014;124(11):E431-6.

28. Imadome K, Iwakawa M, Nakawatari M, Fujita H, Kato S, Ohno T, Nakamura E, Ohkubo Y, Tamaki T, Kiyohara H, et al. Subtypes of cervical adenosquamous carcinomas classified by EpCAM expression related to radiosensitivity. Cancer Biol Ther. 2010;10(10):1019-26.

29. Poczatek RB, Myers RB, Manne U, Oelschlager DK, Weiss HL, Bostwick DG, Grizzle WE. Ep-cam levels in prostatic adenocarcinoma and prostatic intraepithelial neoplasia. J Urol. 1999;162(4):1462-6.

30. Benko G, Spajic B, Kruslin B, Tomas D. Impact of the EpCAM expression on biochemical recurrence-free survival in clinically localized prostate cancer. Urol Oncol. 2011;31(4):468-74.

31. Zellweger T, Ninck C, Bloch M, Mirlacher M, Koivisto PA, Helin HJ, Mihatsch MJ, Gasser TC, Bubendorf L. Expression patterns of potential therapeutic targets in prostate cancer. Int J Cancer. 2005;113(4):619-28.

32. Pourmand G, Ziaee AA, Abedi AR, Mehrsai A, Alavi HA, Ahmadi A, Saadati HR. Role of PTEN gene in progression of prostate cancer. Urol J. 2007;4(2):95-100.

33. Taylor BS, Schultz N, Hieronymus H, Gopalan A, Xiao Y, Carver BS, Arora VK, Kaushik $P$, Cerami E, Reva B, et al. Integrative genomic profiling of human prostate cancer. Cancer Cell. 2010;18(1):11-22.

34. Dubrovska A, Kim S, Salamone RJ, Walker JR, Maira SM, Garcia-Echeverria C, Schultz PG, Reddy VA. The role of PTEN/Akt/PI3K signaling in the maintenance and viability of prostate cancer stem-like cell populations. Proc Natl Acad Sci U S A. 2009;106(1):268-73.

35. Lim M, Chuong CM, Roy-Burman P. PI3K, Erk signaling in BMP7-induced epithelial-mesenchymal transition (EMT) of PC-3 prostate cancer cells in 2and 3-dimensional cultures. Horm Cancer. 2011;2(5):298-309.

36. Mulholland DJ, Kobayashi N, Ruscetti M, Zhi A, Tran LM, Huang J, Gleave M, $\mathrm{Wu} \mathrm{H}$. Pten loss and RAS/MAPK activation cooperate to promote EMT and metastasis initiated from prostate cancer stem/progenitor cells. Cancer Res. 2012:72(7):1878-89.

37. Trzpis M, McLaughlin PM, de Leij LM, Harmsen MC. Epithelial cell adhesion molecule: more than a carcinoma marker and adhesion molecule. Am J Pathol. 2007;171(2):386-95

38. Deng Z, Wu Y, Ma W, Zhang S, Zhang YQ. Adoptive T-cell therapy of prostate cancer targeting the cancer stem cell antigen EpCAM. BMC Immunol. 2015;16(1).

39. Gorin MA, Verdone JE, van der Toom E, Bivalacqua TJ, Allaf ME, Pienta KJ. Circulating tumour cells as biomarkers of prostate, bladder, and kidney cancer. Nat Rev Urol. 2017;14(2):90-7

40. van der Gun BT, Melchers $\sqcup$, Ruiters MH, de Leij LF, McLaughlin PM, Rots MG. EpCAM in carcinogenesis: the good, the bad or the ugly. Carcinogenesis. 2010;31(11):1913-21.

41. Visvader JE, Lindeman GJ. Cancer stem cells: current status and evolving complexities. Cell Stem Cell. 2012;10(6):717-28.

42. Li L, Li Y, Wang L, Wu Z, Ma H, Shao J, Li D, Yu H, Nian W, Wang D. Inhibition of Hes1 enhances lapatinib sensitivity in gastric cancer sphereforming cells. Oncol Lett. 2017;14(4):3989-96.

43. Matsumoto T, Takai A, Eso Y, Kinoshita K, Manabe T, Seno H, Chiba T, Marusawa H. Proliferating EpCAM-positive ductal cells in the inflamed liver give rise to hepatocellular carcinoma. Cancer Res. 2017;77(22):6131-43.

44. Suzuki K, Nakamura K, Kato K, Hamada H, Tsukamoto T. Exploration of target molecules for prostate cancer gene therapy. Prostate. 2007;67(11):1163-73.

45. Kurtz JE, Dufour P. Adecatumumab: an anti-EpCAM monoclonal antibody, from the bench to the bedside. Expert Opin Biol Ther. 2010;10(6):951-8.

46. Moldenhauer G, Salnikov AV, Luttgau S, Herr I, Anderl J, Faulstich H. Therapeutic potential of amanitin-conjugated anti-epithelial cell adhesion molecule monoclonal antibody against pancreatic carcinoma. J Natl Cancer Inst. 2012;104(8):622-34.

47. Salnikov AV, Groth A, Apel A, Kallifatidis G, Beckermann BM, Khamidjanov A, Ryschich E, Buchler MW, Herr I, Moldenhauer G. Targeting of cancer stem cell marker EpCAM by bispecific antibody EpCAMxCD3 inhibits pancreatic carcinoma. J Cell Mol Med. 2009;13(9B):4023-33.

48. Yamashita T, Ji J, Budhu A, Forgues M, Yang W, Wang HY, Jia H, Ye Q, Qin LX, Wauthier E, et al. EpCAM-positive hepatocellular carcinoma cells are tumor-initiating cells with stem/progenitor cell features. Gastroenterology. 2009:136(3):1012-24

49. Shigdar S, Lin J, Yu Y, Pastuovic M, Wei M, Duan W. RNA aptamer against a cancer stem cell marker epithelial cell adhesion molecule. Cancer Sci. 2011; 102(5):991-8. 